\title{
The Use of SWEPT SOURCE OCT ANGIO in Diagnosis and Staging of Type 2 Macular Telangictasia (Mactel 2)
}

\author{
Almamoori Fawwaz* \\ Department of Medical Retina, Eye Specialty Hospital, Jordan
}

Submission: December 06, 2016; Published: December 15, 2016

*Corresponding author: Almamoori Fawwaz, Department of Medical Retina, Eye Specialty Hospital, Amman, Jordan, Tel: 00962777519101; Email: fawazalmamoori@gmail.com

\begin{abstract}
Introduction: Macular telangiectasia type 2 has also been termed idiopathic perifoveal telangiectasia or idiopathic juxtafovealtel angiectasis type 2 [1-4]. It is now referred to as MacTel type 2, and it is a bilateral perifoveal vasculopathy which originates in the deep retinal capillary plexus in the temporal juxtafoveal region. As it progresses, it involves the superficial retinal capillary plexus, and continues to progress anteriorly, posteriorly, and circumferentially. This is called the non-proliferative stage of the disease [5-9]. Mactel type 2 becomes proliferative when the vasogenic process extends under the retina, forming detachment and a retinal-retinal anastomosis. This form of the disease may eventually lead to disciform scarring.
\end{abstract}

Aim \& objectives: Diagnosis and staging of macular telangiectasia type 2 (MacTel2) using Swept Source optical coherence tomography Angiography.

Method: We retrospectively review a $60 \mathrm{Y}$ old patient with bilateral MacTel2 evaluated using a swept source OCT (SSOCT). The patient underwent a comprehensive ocular examination and imaging tests as part of the evaluation of her condition. The imaging tests included color fundus imaging (Topcon, Tokyo, Japan), digital fundus AF imaging, FA\& Swept Source OCT with OCT Angio (TRITON TOPCON OCT). The patient had no any other retinal pathology such as diabetic retinopathy or pathologic myopia and not previously treated with photodynamic therapy (PDT), thermal laser, intravitreal injections, or any retinal surgery. Information about previous medical conditions and ocular treatments was obtained by reviewing the medical charts.

Results: (SS-OCT A) detected abnormal microvasculature in all MacTel2 eyes, predominantly in the middle retinal layer. These vessels correlated well with the FA alterations. The abnormal temporal, juxtafoveal microvasculature in MacTel2 became apparent as the disease progressed and in later stages tended to extend circumferentially, with anastomotic vessels temporally.

Conclusion: we used the SS-OCT Angio technique to investigate eyes with MacTel2 using a TRITON SSOCT. To extract the blood flow information and visualize the microvasculature of the central macula, the central macular microvasculature was visualized better than with FA imaging. In addition, the better visualization of the juxtafoveal microvasculature with SS-OCT Angio may also be due, in part, to the absence of leakage on OCT Angio imaging, and it is this leakage that could obscure the normal vasculature seen on routine FA imaging. By using this ability to extract and visualize these retinal layers in MacTel2 and other diseases, SS-OCT Angio imaging may help facilitate the early diagnosis of disease and provide a better understanding of disease progression and the efficacy of treatments.

Keywords: Macular telangiectasia Type 2; Swept source oct angiography; Fluorescein angiography; Perifoveal vasculopathy

Abbreviations: MacTel 2: Macular Telangiectasia type 2; OCT: Optical Coherence Tomography; SS-OCT Angio: Swept Source OCT Angiography; SD-OCT: Spectral Domain OCT; FA: Fluorescein Angiography; FAF: Fundus Auto Florescence; IS-OS-E: photoreceptor inner segment/outer segment/ellipsoid; GCL+IPL: Ganglion Cell Layer Inner Plexiform Layer; INL + OPL: Inner nuclear layer-Outer Plexiform Layer; ONL + ELM: Outer Nuclear Layer+ External Limiting Membrane; BCVA: Best Corrected Visual Acuity

\section{Introduction}

Macular telangiectasia type 2 has also been termed idiopathic perifoveal telangiectasia or idiopathic juxtafoveal telangiectasis type 2 [1-3]. It is now referred to as MacTel type 2, and it is a bilateral perifoveal vasculopathy which originates in the deep retinal capillary plexus in the temporal juxtafoveal region. As it progresses, it involves the superficial retinal capillary plexus, and continues to progress anteriorly, posteriorly, and circumferentially. This is called the non-proliferative stage of the disease [4-9]. Mactel type 2 becomes proliferative when the vasogenic process extends under the retina, forming detachment and a retinal-retinal anastomosis. This form of the disease may eventually lead to disciform scarring. 
In the early stages of the disease, fluorescein angiography (FA) imaging shows abnormal hyperfluorescence and leakage from the temporal, juxtafoveal capillary plexus, [7] As the disease progresses, the hyperfluorescence and leakage spreads circumferentially around the fovea. While FA provides a definitive diagnosis of MacTel 2, [10] it also involves the intravenous injection of a dye that can result in adverse effects such as nausea or vomiting, and rarely fluorescein can elicit an anaphylactic response [11,12]. Autofluorescence (AF) imaging is also useful in diagnosing MacTel2, [13] Due to the depletion of luteal pigment in the temporal juxtafoveal retina, a relative increase in AF is observed in this region [14-16]. As the disease progresses, luteal pigment is lost circumferentially around the fovea and an increase in the relative hyperfluorescence is observed. In the later stages of the disease, atrophy of the RPE is observed, resulting in decreased AF within the central macula.

Optical coherence tomography (OCT), [17] a noninvasive imaging modality, has revealed structural abnormalities in the inner retina such as retinal cavitation with draping of the internal limiting membrane and abnormalities in the outer retina such as disruption of the photoreceptor inner segment/outer segment/ ellipsoid (IS/OS/E) region that were not previously appreciated by FA or AF imaging [18-25]. OCT imaging has improved the early detection of MacTel 2 by identifying these early subtle changes in retinal anatomy, and OCT has proven to be useful for following these alterations in macular anatomy as the disease progresses to foveal atrophy, the formation of intraretinal pigment plaques, and subretinal neovascularization. With the development of spectral-domain OCT (SDOCT) instruments with increased scanning speeds and high-speed swept-source OCT (SSOCT) instruments, OCT microangiography (OMAG) imaging has emerged as a noninvasive strategy to visualize the retina and choroidal microvasculature without the use of an exogenous intravenous dye injection, [26-38] OMAG is a dynamic strategy capable of providing a three dimensional reconstruction of the perfused microvasculature within the retina and choroid and identifying distinct characteristics of the capillary networks located within different layers of the retina and choroid (see "Swept-Source OCT Angiography of the Retinal Vasculature Using Intensity Differentiation-based Optical Microangiography).

\section{Aim \& Objectives}

To evaluate the central macular microvascular network in patients with maculartelangiectasia type 2 (MacTel2) using Swept Source optical coherence tomography Angiography.

\section{Patient and Method}

We retrospectively review a $60 \mathrm{Y}$ old patient with bilateral MacTel2 evaluated using a swept source OCT (SSOCT). The patient underwent a comprehensive ocular examination and imaging tests as part of the evaluation of her condition. The imaging tests included color fundus imaging (Topcon, Tokyo, Japan), digital fundus AF imaging, FA\& Swept Source OCT with OCT Angio (TRITON TOPCON OCT). The patient had no any other retinal pathology such as diabetic retinopathy or pathologic myopia and not previously treated with photodynamic therapy (PDT), thermal laser, intravitreal injections, or any retinal surgery. Information about previous medical conditions and ocular treatments was obtained by reviewing the medical charts. The retina was segmented into three distinct physiological layers: an inner retinal layer from the ganglion cell layer to the inner plexiform layer (GCL + IPL), a middle retinal layer from the inner nuclear layer to the outer plexiform layer (INL + OPL), and an outer retinal layer from outer nuclear layer to the external limiting membrane (ONL + ELM layer). The microvasculature from the superficial capillary plexus in the inner retina is colored red, the microvasculature from the deep capillary plexus is colored green, and any microvascular structures with flow in the outer retina are colored blue.

\section{Results}

(SS-OCT A) detected abnormal microvasculature in all MacTel2 eyes, predominantly in the middle retinal layer. These vessels correlated well with the FA alterations. The abnormal temporal, juxtafoveal microvasculature in MacTel2 became apparent as the disease progressed and in later stages tended to extend circumferentially, with anastomotic vessels temporally. In our Case, the Right Eye was in Early, Non proliferative MacTel2; best corrected visual acuity (BCVA) in her left eye was $20 / 30$. The horizontal B scan with the retinal flow in different layers represented by colors shows the dilated vessels in the deep retinal capillary plexus found in the middle retinal layer, most pronounced in the region temporal to the fovea as observed in green (Figure 1). With intact IS-OS Junction (Figure 2), Fluorescein angiography shows telangiectatic abnormalities with mild hyperfluorescence and leakage in the temporal juxtafoveal region (Figure 3).
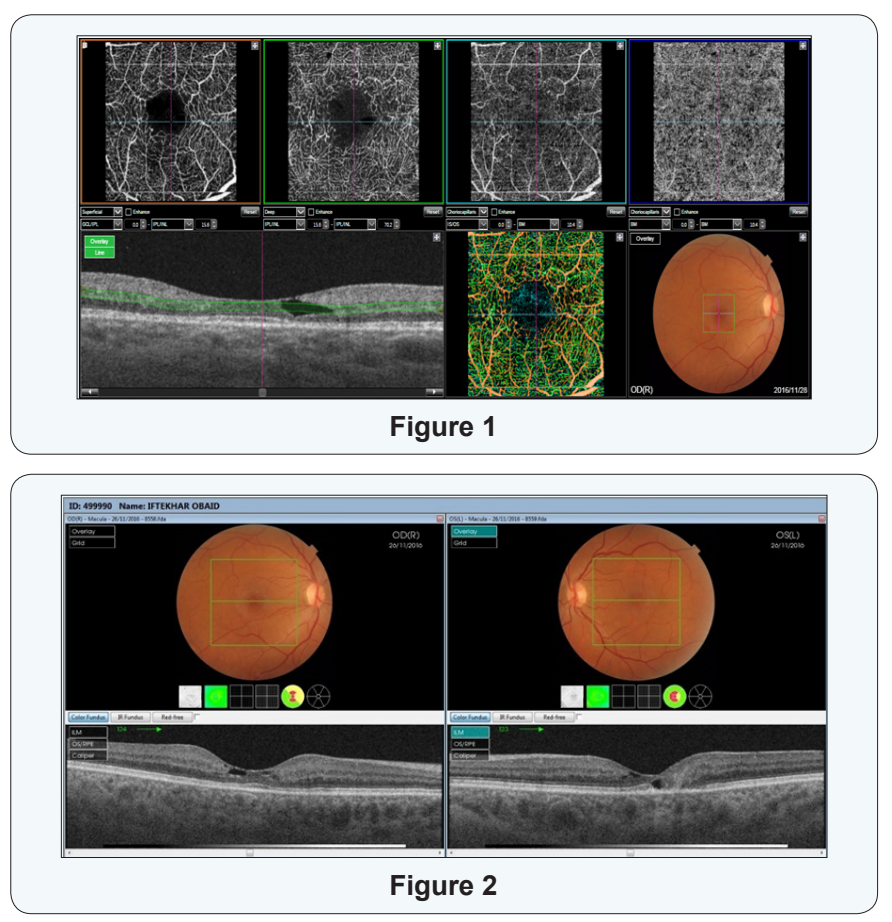


\section{JOJ Ophthalmology}
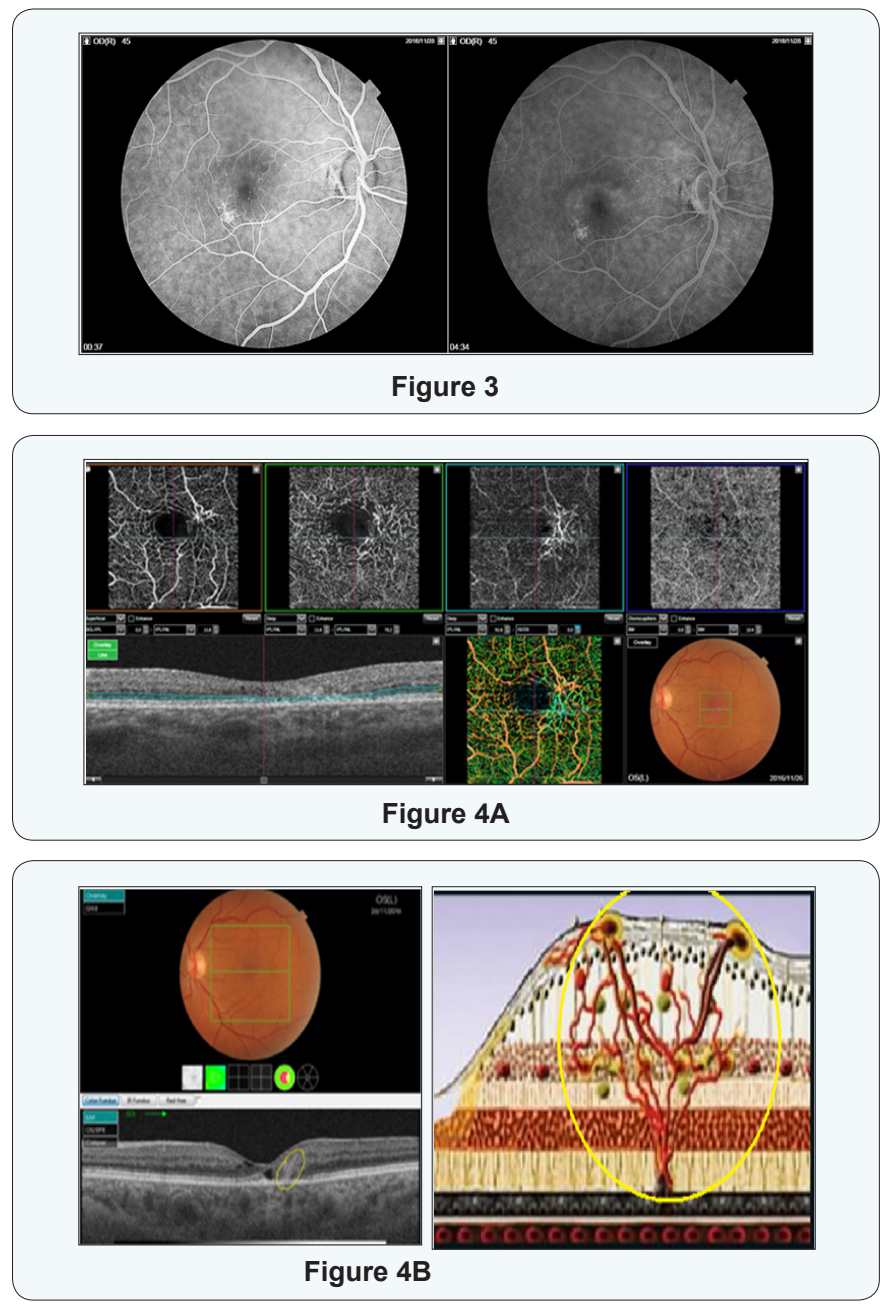

The left eye was in Prolifrative MacTel 2, Best corrected visual acuity (BCVA) in her left eye was 20/50, The Bscan shows cavitation in the outer retina and disruption of the IS/ OS/E boundary in the temporal juxtafoveal region. The Bscan representing the microvascular flow (Figure 4 A\&B) details the presence of abnormal microvasculature (green and blue corresponding to an area with retinal vascular anastomoses. Disruption of the microvasculature extends into the outer retina where the IS/OS/E is disrupted. Microvascular abnormalities, such as a distorted juxtafoveal capillary plexus with prominent anastomoses, FA imaging demonstrates hyperfluorescence in the temporal juxtafoveal region in the earliest stage associated with late leakage (Figure 5)

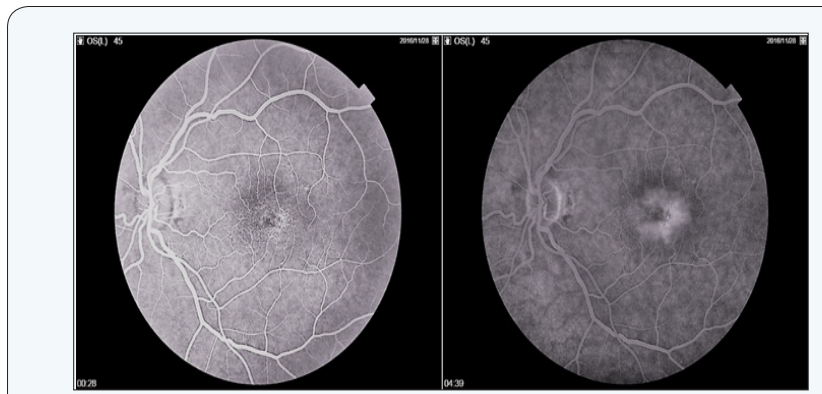

Figure 5

\section{Conclusion}

We used the SS-OCT Angio technique to investigate eyes with MacTel2 using a TRITON SSOCT. To extract the blood flow information and visualize the microvasculature of the central macula, the central macular microvasculature was visualized better than with FA imaging. In addition, the better visualization of the juxtafoveal microvasculature with SS-OCT Angio may also be due, in part, to the absence of leakage on OCT Angio imaging, and it is this leakage that could obscure the normal vasculature seen on routine FA imaging. By using this ability to extract and visualize these retinal layers in MacTel2 and other diseases, SS-OCT Angio imaging may help facilitate the early diagnosis of disease and provide a better understanding of disease progression and the efficacy of treatments and to differentiate MacTel2 from other diseases affecting the retinal microvasculature associated with fluorescein angiographic leakage, such as neovascularage-related macular degeneration, diabetic macular edema, vein occlusions, and cystoid macular edema from differing conditions.

\section{References}

1. Gass JD, Blodi BA (1993) Idiopathic juxtafoveolar retinal telangiectasis. Update ofclassification and followup study. Ophthalmology 100(10): 1536-1546.

2. Gass JD, Oyakawa RT (1982) Idiopathic juxtafoveolar retinal telangiectasis. Arch Ophthalmol 100(5): 769-780.

3. Yannuzzi LA, Bardal AM, Freund KB, Chen KJ, Eandi CM, et al. (2006) Idiopathic macular telangiectasia. Arch Ophthalmol 124(4): 450-460.

4. Yannuzzi LA (2010) The Retinal Atlas. Elsevier, Oxford, Saunders, London.

5. Aung KZ, Wickremasinghe SS, Makeyeva G, Robman L, Guymer RH (2010) The prevalence estimates of macular telangiectasia type 2: the Melbourne Collaborative Cohort Study. Retina 30(3): 473-478.

6. Klein R, Blodi BA, Meuer SM, Myers CE, Chew EY, et al. (2010) The prevalence of macular telangiectasia type2 in the Beaver Dam eye study. Am J Ophthalmol 150(1): 55-62.

7. Clemons TE, Gillies MC, Chew EY, Bird AC, Peto T, et al. (2010) Baseline characteristics of participants in the natural history study of macular telangiectasia (MacTel) MacTel Project Report No. 2. Ophthalmic Epidemiol 17(1): 66-73.

8. Thorell MR, Zhang Q Huang Y, An L, Durbin MK (2014) SweptSource OCT Angiography of Macular Telangiectasia Type 2. Ophthalmic Surg Lasers Imaging Retina 45(5): 369-380.

9. Sallo FB, Leung I, Chung M, Wolf-Schnurrbusch UE, Dubra A, et al. (2011) Retinal crystals in type 2 idiopathic macular telangiectasia. Ophthalmology 118(12): 2461-2467.

10. Zhu M, Krilis M, Gillies MC (2013) The relationship between inner retinal cavitation, photoreceptor disruption, and the integrity of the outer limiting membrane in macular telangiectasia type 2 . Retina 33(8): 1547-1550.

11. SchmitzValckenberg S, Ong EE, Rubin GS, Peto T, Tufail A, et al. (2009) Structural and functional changes over time in MacTel patients. Retina 29(9): 1314-1320.

12. LopezSaez MP, Ordoqui E, Tornero P, Baeza A, Sainza T, et al. (1998) Fluoresceininduced allergic reaction. Ann Allergy Asthma Immunol 81(5): 428-430. 
13. Lira RP, Oliveira CL, Marques MV, Silva AR, Pessoa Cde C, et al. (2007) Adverse reactions of fluorescein angiography: a prospective study. Arq Bras Oftalmol 70(4): 615-618.

14. Charbel Issa P, Gillies MC, Chew EY, Bird AC, Heeren TF, et al. (2013) Macular telangiectasia type 2. Prog Retin Eye Res 34: 49-77.

15. Theelen T, Berendschot TT, Boon CJ, Hoyng CB, Klevering BJ (2008) Analysis of visual pigment by fundus autofluorescence. Exp Eye Res 86(2): 296-304.

16. Wong WT, Forooghian F, Majumdar Z, Bonner RF, Cunningham D, et al. (2009) Fundus autofluorescence in type 2 idiopathic macular telangiectasia: correlation with optical coherence tomography andmicroperimetry. Am J Ophthalmol 148(4): 573-583.

17. Ooto S, Hangai M, Takayama K, Arakawa N, Tsujikawa A, et al. (2011) Highresolution photoreceptor imaging in idiopathic macular telangiectasia type 2 using adaptive optics scanning laser ophthalmoscopy. Invest Ophthalmol Vis Sci 52(8): 5541-5550.

18. Balaskas K, Leung I, Sallo FB, Clemons TE, Bird AC, et al. (2014) Associations between aautofluorescence abnormalities and visual acuity in idiopathic macular telangiectasia type 2: MacTel Project report number 5. Retina 34(8): 1630-1636.

19. Mathew R, Sivaprasad S, Florea D, Leung I, Sallo F, et al. (2013) Agreementbetween timedomain and spectraldomain optical coherence tomography in the assessment of macular thickness in patients with idiopathic macular telangiectasia type 2. Ophthalmologica 230(3): 144-150.

20. Surguch V, Gamulescu MA, Gabel VP (2007) Optical coherence tomography findings in idiopathicjuxtafoveal retinal telangiectasis. Graefes Arch Clin Exp Ophthalmol 245(6): 783-788.

21. Gaudric A, Ducos de Lahitte G, Cohen SY, Massin P, Haouchine B (2006) Optical coherence tomography in group 2A idiopathic juxtafoveolar retinal telangiectasis. Arch Ophthalmol 124(10): 1410-1419.

22. Paunescu LA, Ko TH, Duker JS, Chan A, Drexler W, et al. (2006) Idiopathic juxtafoveal retinal telangiectasis: new findings by ultrahighresolution optical coherence tomography. Ophthalmology 113(1): 4857.

23. Koizumi H, Iida T, Maruko I (2006) Morphologic features of group 2A idiopathic juxtafoveolar retinal telangiectasis in threedimensional optical coherence tomography. Am J Ophthalmol 142(2): 340-343.

24. Baumuller S, CharbelIssa P, Scholl HP, Schmitz-Valckenberg S, Holz FG (2010) Outer retinal hyperreflective spots on spectraldomain optical coherence tomography in macular telangiectasia type 2 . Ophthalmology 117(11): 2162-2168.

25. Sallo FB, Peto T, Egan C, Wolf-Schnurrbusch UE, Clemons TE, et al. (2012) The IS/OS junction layer in the natural history of type 2 idiopathic macular telangiectasia. Invest Ophthalmol Vis Sci 53(12): 7889-7895.

26. Sallo FB, Peto T, Egan C, Wolf-Schnurrbusch UE, Clemons TE, et al. (2012) "En face" OCT imaging of the IS/OS junction line in type 2 idiopathic macular telangiectasia. Invest Ophthalmol Vis Sci 53(10): 6145-6152.

27. Wang RK, An L, Francis P, Wilson DJ (2010) Depthresolved imaging of capillary networks in retina and choroid using ultrahigh sensitive optical microangiography. Opt Lett 35(9): 1467-1469.

28. An L, Johnstone M, Wang RK (2012) Optical microangiography provides correlation between microstructure and microvasculature of optic nerve head in human subjects. J Biomed Opt 17(11): 116018.

29. Wang RK (2010) Optical Microangiography: A label free 3d imaging technology to visualize and quantify blood circulations within tissue beds in vivo. IEEE J Sel Top Quantum Electron 16(3): 545-554.

30. Wang RK, Jacques SL, Ma Z, Hurst S, Hanson SR, et al (2007) Three dimensional optical angiography. Opt Express. 15(7): 4083-4097.

31. Choi W, Mohler KJ, Potsaid B, Lu CD, Liu JJ, et al. (2013) Choriocapillaris and choroidal microvasculature imaging with ultrahigh speed OCT angiography. PLoS One 8(12): e81499.

32. JiaY,BaileyST,WilsonDJ,Tan O, Klein ML,etal. (2014) Quantitative optical coherence tomography angiography of choroidal neovascularization in agerelated macular degeneration. Ophthalmology 121(7): 1435-1444.

33. Wei E, Jia Y, Tan O, Potsaid B, Liu JJ, et al. (2013) Parafoveal retinal vascular response to pattern visual stimulation assessed with OCT angiography. PLoS One 8(12): e81343.

34. Braaf B, Vermeer KA, Vienola KV, de Boer JF (2012) Angiography of the retina and the choroid with phaseresolved OCT using intervaloptimized backstitched Bscans. Opt Express. 20(18): 20516-20534.

35. Schwartz DM, Fingler J, Kim DY, Zawadzki RJ4, Morse LS, et al. (2014) Phasevariance optical coherence tomography: a technique for noninvasive angiography. Ophthalmology 121(1): 180-187.

36. Kim DY, Fingler J, Zawadzki RJ, Susanna S Parkb, Lawrence S Morseb, et al. (2013) Optical imaging of the chorioretinal vasculature in the living human eye. Proc Natl Acad Sci USA 110(35): 14354-14359.

37. Motaghiannezam SM, Koos D, Fraser SE (2012) Differential phasecontrast, sweptsource optical coherence tomography at $1060 \mathrm{~nm}$ for in vivo human retinal and choroidal vasculature visualization. J Biomed Opt 17(2): 026011.

38. Kim DY, Fingler J, Zawadzki RJ, Park SS, Morse LS, et al. Noninvasive imaging of the foveal avascular zone with high-speed, phase-variance optical coherence tomography. Invest Ophthalmol Vis Sci 53(1): 85-92.

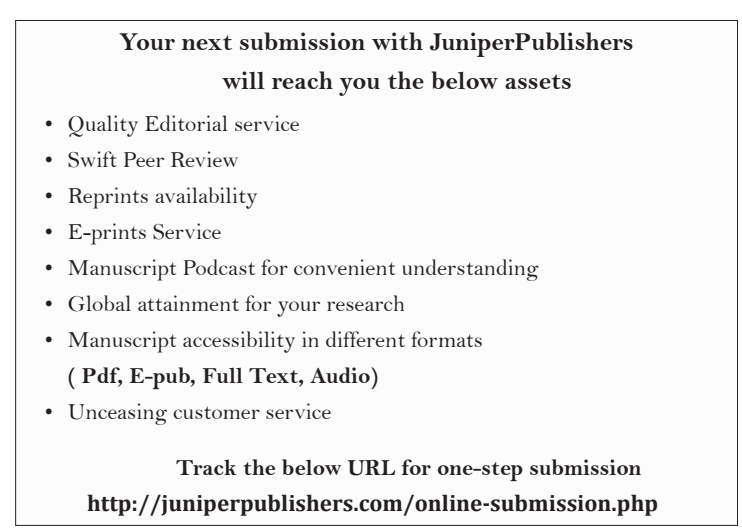

